\section{Mutual coupling reduction in multilayer patch antennas via meander line parasites}

\author{
S. Maddio, G. Pelosi, M. Righini, S. Selleri ${ }^{\bowtie}$ and I. Vecchi
}

A method to reduce the mutual coupling in wide-band multilayer patch array operating in $\mathrm{C}$ band is presented. The proposed device use conveniently sized meander line parasites printed on the same substrate of the upper patch. Measurements on a $2 \times 2$ prototype shows a measured bandwidth of about $19.6 \%$ centred at $5.1 \mathrm{GHz}$, therefore adequate to cover the whole WLAN frequency range. The meander lines allow to reduce the patch mutual coupling by about $2.4 \mathrm{~dB}$.

Introduction: Mutual coupling (MC) is an unavoidable phenomenon occurring among neighbouring antennas, which is particularly critical when antennas are in array configuration. MC is mainly due to three effects: near field coupling, far filed coupling and surface wave coupling [1]. The first one is dominant when each element is exposed to the near field of its neighbours, while the far field coupling is caused from the radiation in horizontal direction, which is lower when a low-permettivity substrate is used. The third one, finally, is due to the presence of waves bound to the air-dielectric interface and produces an undesired end-fire radiation. Although these effects can be reduced by increasing the distance between the elements, it is necessary to take into account that beyond a certain distance undesired grating lobes appear. This issue is critical in active electronically scanned array application, since the maximum distance between elements must be minimised in order to maximise scanning ability.

Several methods have been proposed in order to reduce the MC effects in an array. In [2, 3], a defected ground plane structure was used while in [4] an electromagnetic bandgap structure is introduced. Although both methods show an effective MC reduction, they both lead to narrow band systems and increased manufacturing complexity. Active cancellation is possible, in principle [5], but is even more complex and costly. A more viable, fully passive, technique, presented in [6], exploits printed U-shaped microstripline parasites. Since the parasite is printed on the same layer of the radiating elements, the structure complexity does not increase. In [6], anyway elements and parasites are narrow-banded.

In this Letter, a technique similar to the one presented in [6] is used, but for a multilayer wide-band patch array operating in $\mathrm{C}$ band over a $19.6 \%$ bandwidth. The parasite is a meander line designed for minimal impact on radiation characteristics and production costs, yet allowing $\mathrm{MC}$ reduction.

Antenna design: A prototype array comprising four multilayer patch elements composed of two FR408 $\left(\epsilon_{r}=3.67, \tan \delta=0.012\right)$ layers separated by a $3.7 \mathrm{~mm}$ air gap, realised employing four nylon spacers, bolts and nuts.

The single element (Fig. 1) is composed of two patches: patch $P_{1} \quad\left(W_{1}=11.4 \mathrm{~mm}, L_{1}=15.2 \mathrm{~mm}\right)$, which is directly fed (coaxial probe $d_{f}=2.1 \mathrm{~mm}$ from shortest side), is etched over the lower substrate; patch $P_{2}\left(W_{2}=12.4 \mathrm{~mm}, L_{2}=15.69 \mathrm{~mm}\right)$ is etched over the upper substrate and centred on the first.

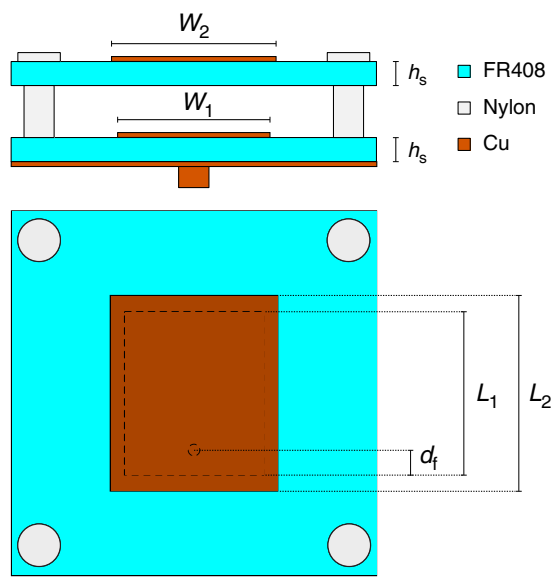

Fig. 1 Layout of the single patch antenna element
Each element is fed by a $50 \Omega$ coaxial cable, whose core is solder to the lower patch, while the shield is solder to the ground plane. The choice of air as an intermediate layer, with respect to a fully solid structure [7], allows to increase the bandwidth performances without adding weight [8]. Unfortunately, the presence of the air requires larger patches for a given frequency, since patch length is proportional to half wavelength in the layered medium. Since the distance between the patches phase centres must be kept close to half wavelength in air to maximise scanning ability, larger patches lead automatically to closer patch borders in the array configuration and hence a sensible increase in MC

Mutual coupling: To reduce the MC microstrip meander lines are printed on the upper substrate (Fig. 2). These lines couple with the neighbouring patches, effectively producing a second coupling path ( $2^{\circ} \mathrm{MC}$ path) which adds to the original and unavoidable coupling path $\left(1^{\circ} \mathrm{MC}\right.$ path), as shown in Fig. 2. If the meander lines are opportunely sized the second MC path can be made to be in phase opposition with respect to the first one and close to it in magnitude, so that global $\mathrm{MC}$ tends to vanish. The line is placed where MC is most critical, in this case (Fig. 2) between the two radiating edges of neighbouring patches aligned along their $E$-plane.

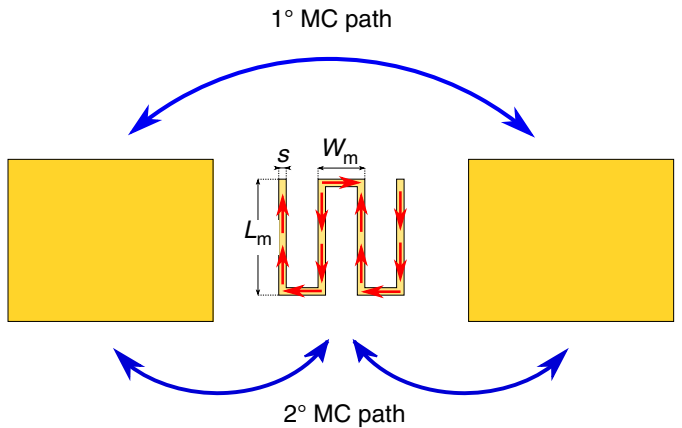

Fig. $2 M C$ path considering the microstrip meander line, with meander dimensions and induced currents. Patches radiating edges are the vertical ones

The meander line has been chosen in order to minimise its effects on the radiation pattern. Its induced currents exhibit a global mutual cancellation of their far field components due to the repeated change in direction, hence the radiation performances of the array are little affected (Fig. 2). Meander line dimensions are: $L_{m}=6.2 \mathrm{~mm}$, $W_{m}=2.5 \mathrm{~mm}$ and $s=0.2 \mathrm{~mm}$.

Simulation and measurement results: A $2 \times 2$ prototype has been fabricated, and is shown in Fig. 3. The array is realised via photo-chemical etching and fed via four SMA connectors.
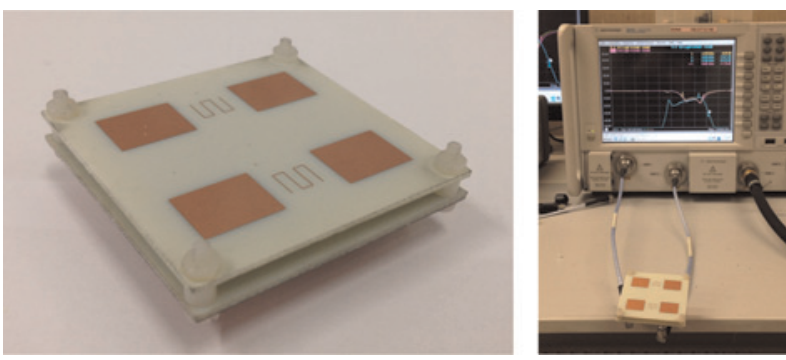

Fig. $32 \times 2$ array prototipe, with meander line parasites

In order to check the MC reduction, a second structure, with identical patches but without parasites has been prepared. To minimise prototype differences due to soldering and assembling tolerances, only the upper layer is duplicated, so that measurements have been carried out on the same lower layer, with different upper layers.

A comparison between simulations and measurements is shown below. Simulations have been carried out using the full-wave software CST studio suite while measurements have been made using a Keysight 5242A Vector Network Analyser (20 MHz-26 GHz).

Fig. 4 shows the comparison between the simulated and the measured reflection coefficients. Due to the symmetry of the structure, only one 
port is presented here, so these results are expressed in terms of $\left|S_{1,1}\right|$. The green lines represent the response of the reference structure, without parasites, while the blue ones show the performances with the meander lines. In both cases, a shift of the central frequency of about $200 \mathrm{MHz}$ is present, which is due to the tolerance on the FR408 dielectric constant [7], and on the spacers. Anyway, the measurements behaviour reflects the simulations one and only a small reduction of the bandwidth due to the presence of the meander lines is observed. Considering a $\mathrm{RL}>10 \mathrm{~dB}$, the simulated bandwidth of the structure without parasites is $21.3 \%$, while the array with parasites shows a $19.84 \%$ value. For measurements the percentage bandwidth are 22.8 and $21.3 \%$, respectively. In both cases, the percentage bandwidth of the designed array covers the whole WLAN frequency range.

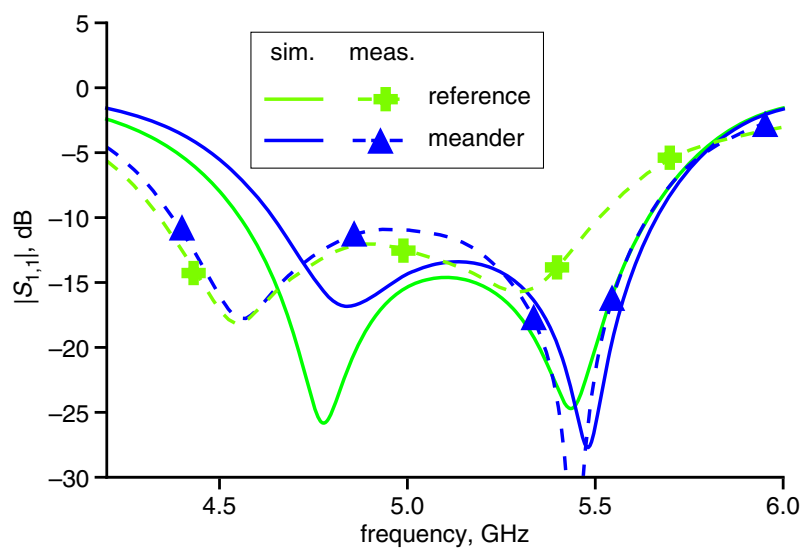

Fig. 4 Measured (dashed line) and simulated (solid line) reflection coefficients. The reference structure results are plotted in green while the meander structure results are plotted in blue

Fig. 5 shows the comparison between the simulated and the measured transmission coefficients, that is the MC levels. Since the analysis on the reference structure showed the highest MC value on the $E$-plane, only this worst case is reported. Despite the presence of the already mentioned frequency shift, also in this case a good agreement between measurement and simulation general behaviour is apparent. Considering the simulation results the worst case for the basic structure shows a MC of $-11.76 \mathrm{~dB}$ at $4.57 \mathrm{GHz}$, while the one for the proposed array is $-13.68 \mathrm{~dB}$ at $5.25 \mathrm{GHz}$. That is nearly $2 \mathrm{~dB}$ better. Measured $\mathrm{MC}$ drops from $-13.109 \mathrm{~dB}$ at $5.21 \mathrm{GHz}$ to $-15.51 \mathrm{~dB}$ at $5.29 \mathrm{GHz}$, for the array without and with parasites, respectively. In this case, the decrease is even better and equal to $2.4 \mathrm{~dB}$.

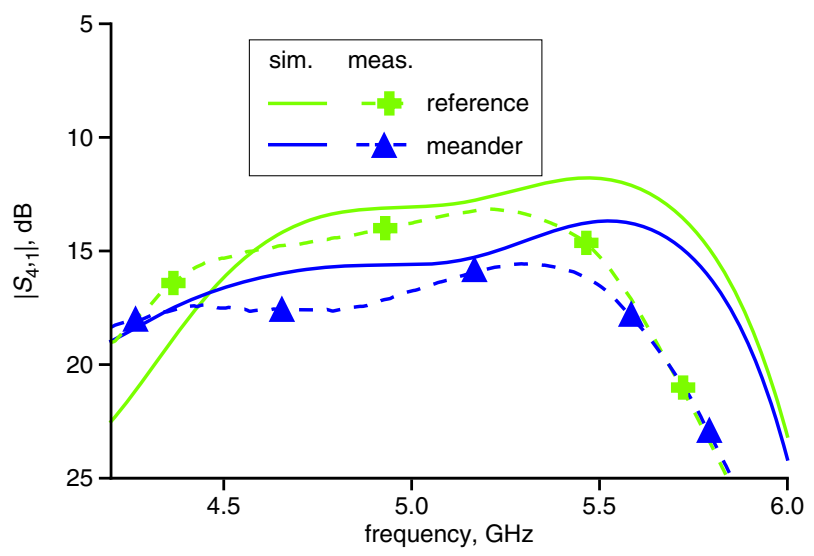

Fig. 5 Measured (dashed line) and simulated (solid line) MC. The reference structure results are plotted in green while the meander structure results are plotted in blue

Finally, a comparison between simulated and measured patterns for both structures at $5.8 \mathrm{GHz}$ is presented in Fig. 6. Measurements have been carried out in a semi-anechoic chamber. It is apparent how the presence of the meander lines does not affect the pattern shape, since simulations and measures are nearly superimposed for both structures.

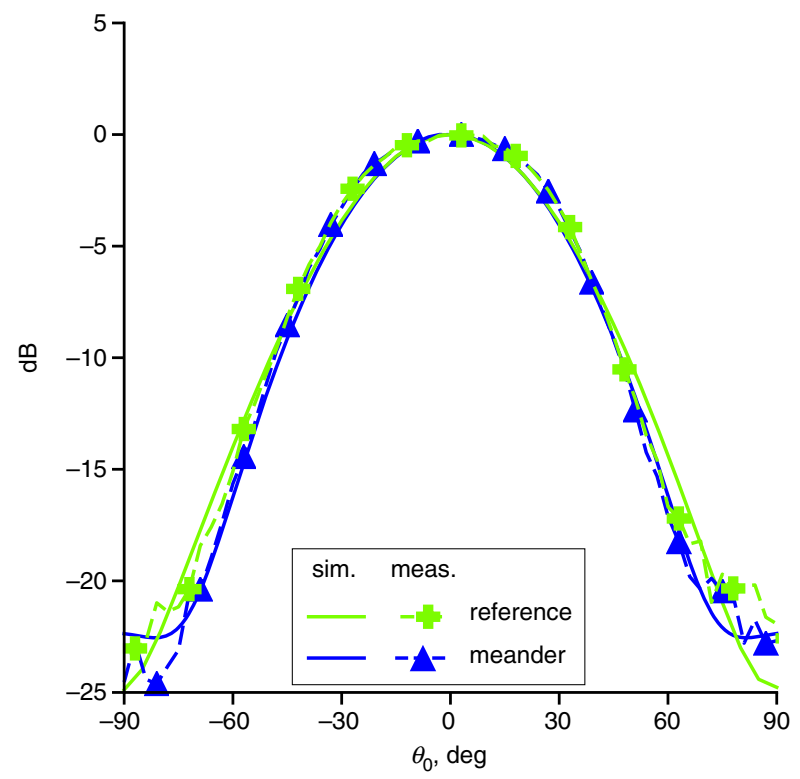

Fig. 6 Measured (dashed line) and simulated (solid line) patterns at $5.8 \mathrm{GHz}$. The reference structure results are plotted in green while the meander structure results are plotted in blue

Conclusion: A technique to reduce the $\mathrm{MC}$ for wide band multilayer patch arrays and experimentally proven on a $2 \times 2$ array working at $5.1 \mathrm{GHz}$ has been shown. This method is based on the addition of two meander microstrip lines on the same substrate of the upper patch. Although their presence slightly reduces the measured bandwidth the resulting value, $21.3 \%$, is anyway sufficient to cover the whole WLAN frequency range. In addition, a reduction of about $2.4 \mathrm{~dB}$ in the MC is shown. A comparison between the two structures in terms of pattern shows that the presence of the meander lines does not affect the antenna pattern.

(C) The Institution of Engineering and Technology 2018

Submitted: 19 April 2018 E-first: 12 June 2018

doi: $10.1049 / \mathrm{el} .2018 .1332$

One or more of the Figures in this Letter are available in colour online.

S. Maddio, G. Pelosi, M. Righini, S. Selleri and I. Vecchi (DINFO, University of Florence, Florence, Italy)

凶-mail: stefano.selleri@unifi.it

\section{References}

1 Nicolić, M.M., Djordjević, A.R., and Nehorai, A.: 'Microstrip antennas with suppressed radiation in horizontal directions and reduced coupling', Trans. Antennas Propag., 2005, 53, pp. 3469-3476

2 Bait-Suwailam, M.M., Siddiqui, O.F., and Ramahi, O.M.: 'Mutual coupling reduction between microstrip patch antennas using slottedcomplementary split-ring resonators', Antennas Wirel. Propag. Lett., 2010, 9, pp. 876-878

3 Wei, K., Li, J.Y., Wang, L., et al.: 'Mutual coupling reduction by novel fractal defected ground structure bandgap filter', Trans. Antennas Propag., 2016, 64, pp. 4328-4335

4 Rajo-Iglesias, E., Quevedo-Teruel, Ó., and Inclán-Sánchez, L.: 'Mutual coupling reduction in patch antenna arrays using a planar EBG structure and a multilayer dielectric substrate', Trans. Antennas Propag., 2008, 11, pp. 1648-1655

5 Maddio, S., Cidronali, A., and Manes, G.: 'Real-time adaptive transmitter leakage cancelling in 5.8-GHz full-duplex transceivers', Trans. Microw. Theory Tech., 2015, 63, pp. 509-519

6 Farsi, S., Aliakbarian, H., Schreurs, D., et al.: 'Mutual coupling reduction between planar antennas by using a simple microstrip U-section', Antennas Wirel. Propag. Lett., 2012, 11, pp. 1501-1503

7 Giunta, G., Novi, C., Maddio, S., et al.: 'Efficient tolerance analysis on low cost, compact size, wideband multilayer patch antenna'. IEEE APS Symp., San Diego, California, USA, 9-14 July 2017, pp. 2113-2114

8 Capece, P., Lucci, L., Pelosi, G., et al.: 'A multilayer PCB dual polarized radiating element for future radar applications', Antennas Wirel. Propag. Lett., 2014, 13, pp. 297-300 